Acquisition et interaction en langue étrangère

16 | 2002

L'Acquisition en classe de langue

\title{
La classe de langue un lieu ordinaire, une interaction complexe
}

\section{Francine Cicurel}

\section{(2) OpenEdition \\ 1 Journals}

Édition électronique

URL : https://journals.openedition.org/aile/801

DOI : 10.4000/aile.801

ISSN : $1778-7432$

Éditeur

Association Encrages

Édition imprimée

Date de publication : 2 septembre 2002

Pagination : 145-164

ISSN : 1243-969X

\section{Référence électronique}

Francine Cicurel, «La classe de langue un lieu ordinaire, une interaction complexe », Acquisition et interaction en langue étrangère [En ligne], 16 | 2002, mis en ligne le 14 décembre 2005, consulté le 14 avril 2022. URL : http://journals.openedition.org/aile/801 ; DOI : https://doi.org/10.4000/aile.801

Ce document a été généré automatiquement le 14 avril 2022.

(c) Tous droits réservés 


\title{
La classe de langue un lieu ordinaire, une interaction complexe
}

\author{
Francine Cicurel
}

1 Une langue étrangère peut être apprise dans de multiples situations - milieu familial, séjours linguistiques dans le pays de la langue-cible, autodidaxie, nouvelles technologies, etc. - mais force est de constater que c'est le plus souvent une institution éducative qui est en charge de l'enseignement/apprentissage des langues. Le dispositif éducatif produit lui-même des discours traitant de la transmission de la discipline en question (Ali Bouacha 1984, Moirand 1986). Les instructions officielles, les programmes, les manuels contiennent des traces discursives permettant de voir quelle est l'appropriation visée. L'institution se charge de mesurer, évaluer, apprécier, voire consigner les performances des impétrants. Mais qu'est-ce qui est évalué ? les progrès dans la langue ou la capacité à effectuer les activités que l'institution propose ? Les recherches en acquisition des langues étrangères se portent aujourd'hui sur l'apprentissage en classe ; elles rencontrent la complexité du dispositif institutionnel, à l'origine de production de textes prescriptifs, de mise en œuvre de programmes, de découpage de la matière enseignée, d'évaluation institutionnelle, ce qui rend difficile l'isolement des facteurs individuels liés à l'appropriation. À l'heure actuelle, on ne dispose pas de résultats globaux permettant de comparer les trajectoires et les progressions des individus en fonction des méthodes, moyens, supports, discours instructionnel; on voit plutôt l'émergence d'études partielles, qui prennent pour cible des fragments du processus acquisitionnel et des descriptions assez fines des contextes de classe et des pratiques langagières qui leur sont propres (voir le Xe Colloque Acquisition d'une langue étrangère: perspectives et recherches en 1996 à Besançon, Souchon 1998, le XIIe Colloque Usages pragmatiques et acquisition des langues à Paris, 1999, à paraître, Les carnets du Cediscor 4 et 7). Nombreux et complexes sont les facteurs entrant en ligne de compte dans une situation d'apprentissage en classe et, face à la multiplicité des paramètres, il s'avère difficile de dégager des liens de causalité entre tel aspect du processus instructionnel et le processus d'apprentissage.

1. Regards sur la classe de langue comme objet de recherche, lieu de formation et d'interaction 
2 L'intérêt dans le domaine de la didactique des langues pour la classe comme lieu privilégié de l'apprentissage ou de l'enseignement est ancien, et légitime, car c'est bien là que s'effectue la rencontre entre le pôle enseignant, le pôle apprenant et la matière à apprendre. Si aujourd'hui la pratique de terrain propre aux analystes de la conversation a rendu familières les consignations des événements de la classe grâce aux enregistrements et aux transcriptions, le regard que l'on porte sur ce lieu d'enseignement/ apprentissage et sur ce qu'on attend de lui s'est modifié : les attentes, les représentations, le rôle que l'on fait jouer à la classe ne sont plus les mêmes.

1.1. La classe de langue comme lieu d'une pratique exemplaire

3 Dans les années 60-70, les classes deviennent, dans certains contextes, des lieux de réalisation de méthodologies nouvelles, supposées performantes. On a introduit aux États-Unis la méthode audio-linguale et en France les méthodes structuro-globales audiovisuelles, méthodes qui veulent développer en priorité les compétences orales : la classe devient alors un lieu d'actualisation d'une méthodologie dominante. Les «moments de la classe» des méthodes audiovisuelles constituent des étapes obligées. Cette époque glorieuse, marquée par la conviction de pouvoir valider scientifiquement la mise en place d'une méthode d'enseignement universellement performante est suivie dix ou quinze ans plus tard d'un grand scepticisme (voir la description faite par Coste, 1986 des trois décennies depuis les années 60). Le Pennsylvania Project (Smith, 1970), comparant trois méthodes différentes d'enseignement du français et de l'allemand à des élèves de niveaux débutant et intermédiaire, démontre que n'est pas suffisant l'écart entre les résultats pour qu'une méthodologie puisse être considérée comme meilleure qu'une autre. En France, dès 1974, un article de Moirand à propos du type de communication généré par les méthodes audiovisuelles évoque le "désenchantement» et marque le début d'une déconstruction de l'hégémonie méthodologique.

1.2. La classe de langue comme lieu de formation

4 Assister à des classes fait partie de la plupart, sinon de la totalité, des programmes de formation pédagogique. Afin de ne pas laisser place à des attitudes subjectives, des observations systématiques avec recours à des grilles, qui permettent de noter les événements de la classe, sont utilisées. Ainsi, la célèbre grille de Flanders (1960) a pour objet d'évaluer le comportement d'un professeur, sa directivité ou son autoritarisme, selon qu'il laisse plus ou moins place à l'initiative de l'apprenant, qu'il l'encourage, qu'il le félicite, etc. Sur les dix entrées proposées par la grille, seules deux sont consacrées à l'intervention verbale des apprenants. Malgré cela, il s'agit d'un incontestable tournant: la classe devient le lieu de l'interaction plutôt que la réalisation d'une méthodologie idéale. On voit également l'émergence d'études portant sur des journaux de bord ou journaux d'apprentissage, dans lesquels sont consignés les événements d'apprentissage et le vécu durant un cours de langue (voir les travaux de Grandcolas et Bailey). En France, dans les années 80, les filières Français Langue Étrangère intègrent systématiquement dans leur cursus la rédaction d'un journal d'apprentissage, qui permet aux étudiants de constituer des données rétrospectives sur les conditions d'appropriation d'une langue éloignée de leur langue maternelle, et sur la façon dont cette appropriation est vécue. L'observable - différé, passant par le filtre de la mémoire du scripteur - n'est pas seulement la vie immédiate de la classe, mais relève aussi d'une approche réflexive.

1.3. La classe de langue, lieu d'une interaction 
5 Le développement des recherches sur la conversation dans le domaine de la pragmatique interactionnelle pousse à considérer la classe, à partir des années 80 , comme un lieu socialisé, où s'établit un échange actif entre des partenaires ayant leur place dans l'interaction. Les interactants ont des buts partiellement convergents (à visée didactique), préexistant à l'interaction et la légitimant (programmes, objectifs à atteindre, résultats), mais cette planification n'élimine pas pour autant l'existence de dispositifs communicationnels complexes. Constamment, l'action planifiée du professeur rencontre des épisodes pouvant survenir dans le déroulement de l'interaction et la modifier. Dans une perspective alors plus proche de l'approche ethnométhodologique (voir la présentation faite par Mondada, 2000), le regard de l'analyste se porte alors sur les méthodes mises en place par les interactants ${ }^{1}$ - aussi bien le participant-expert (dont la place exige qu'il fasse appel à diverses stratégies pour se faire comprendre, pour favoriser l'apprentissage, pour attirer l'attention sur les problèmes langagiers, etc.) que les participants-apprenants co-produisant le discours avec l'enseignant. S'il faut se garder de généraliser les résultats d'observations faites sur quelques heures de cours, il n'en reste pas moins que la constitution de corpus à partir de transcriptions d'interactions en classe de langue ${ }^{2}$ permet de mieux cerner un certain nombre de points :

6 - la place des participants, notamment celle de l'apprenant qui, par des marques intonatives, des marques d'hésitation, rappelle son statut d'individu en train d'apprendre (et peut-être, à ce titre, ayant droit à l'indulgence) ;

7 - le système d'alternance des tours de parole, qui met en exergue le rôle de l'enseignant comme l'interactant qui intervient à la suite de la plupart des prises de parole des apprenants ;

8 - un format interactionnel prévisible montrant que l'on encourage la production verbale (ce but est parfois tellement dominant qu'il écrase les règles conversationnelles des échanges ordinaires) ;

9 - une activité tournée vers l'amélioration de la compétence langagière, se traduisant pour l'apprenant par des reprises, des reformulations, des hésitations, etc., et pour l'enseignant par le recours à des stratégies comme le guidage, l'étayage, les instructions, les réparations, les explications ;

10 - la dimension métalinguistique manifestée, par exemple, par la fréquence de séquences de focalisation sur le code (voir la bifocalisation décrite par Bange, 1992).

11 - l'instauration d'un contrat de fiction lorsqu'il y a nécessité de configurer des univers imaginaires pour faire vivre la langue ;

12 - l'usage de soi, qui perce sous les rôles assignés par le cadre interactionnel, et qui n'étouffe pas - loin de là - la dimension de l'affect dans l'échange.

13 L'accent n'est plus mis sur les seuls actes d'enseignement mais sur l'interaction dans sa dimension collective, sur le groupe d'apprenants et sur le déroulement de leurs échanges. L'apprenant est vu comme appartenant à un groupe social dans lequel il se fond. Cependant ce rééquilibrage ne va pas tant dans la direction d'un effort pour envisager la classe comme lieu de progression langagière, que pour l'instaurer comme lieu de parole et de socialisation. Dans la lignée des travaux de Mehan (1979) qui, sur la durée d'une année, observe la manière dont de jeunes enfants sont actifs dans l'interaction en classe et dans la gestion de la parole, des études de cas, s'attachant à observer l'évolution d'un apprenant dans un environnement institutionnel donné, 
permettraient d'améliorer la connaissance des comportements langagiers et interactionnels d'un sujet apprenant (voir les travaux présentés dans AILE 4, consacré aux Profils d'apprenants). Procéder à des inventaires de comportements verbaux d'un apprenant (A-t-il des initiatives? Négocie-t-il les réponses? Interagit-il avec les autres ou seulement avec le professeur? Fait-il des réponses minimales? Ses réponses portent-elles sur le contenu proposé ou sur autre chose? Combien de temps tient-il dans l'interaction? Comment réagit-il aux corrections ? etc.) donnerait des indications sur une partie du processus d'apprentissage en contexte scolaire (car l'appropriation ne se réalise pas seulement pendant la durée du cours mais aussi à l'extérieur de la classe, dans les intervalles des rencontres scolaires). L'exposition discursive en classe permet à chaque apprenant d'en faire un usage personnel, et c'est la diversité des comportements qu'il serait intéressant de mieux comprendre.

2. Qu'est-ce que guider une appropriation?

Il suffit d'entrer dans une classe pour le constater, en voyant la disposition de l'espace ou le déroulement interactionnel et ses routines : apprendre une langue en classe ne ressemble nullement à une appropriation langagière en milieu naturel. Enseigner ou apprendre une langue dans une situation de classe peut être représenté par le schéma suivant :

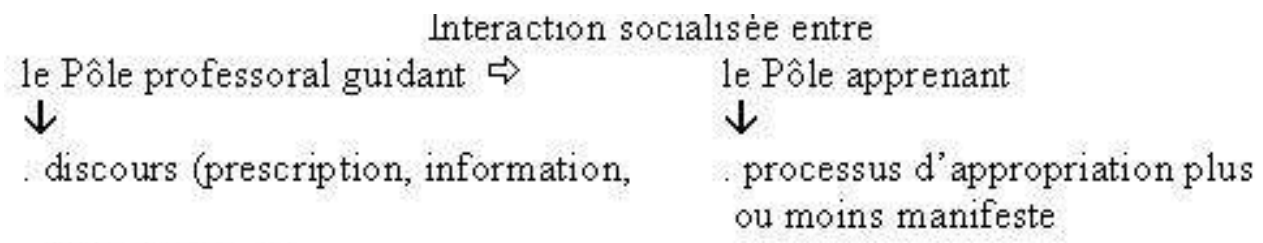

évaluation...)

exigences institutionnelles, méthodologies, conceptions

. activités interactionnelles

de l'apprentissage,...

schéma d'action, interaction

stratégies d'apprentis sage
$\downarrow \downarrow$

par la médiation d'objets (souvent scripturaux: textes, livres, outils, inscriptions au tableau, notes)

$\Rightarrow$ influence du contexte, géographique, institutionnel, du hors classe (statut de la langue apprise,possibilités d'entendre la langue, de la parler, etc.), de la langue des élèves.

D'importants progrès dans la compréhension et la description de ce qui se passe lorsque des locuteurs ayant une connaissance inégale de la langue conversent ont été faits grâce aux linguistes spécialistes de la communication exolingue. Les recherches sur la classe de langue ont bénéficié de ces acquis, car l'échange co-construit au sein de la classe voit l'émergence de phénomènes langagiers comme les demandes d'aide, la reformulation, le contrat didactique, l'hétéro-achèvement, etc. Les travaux de ces chercheurs montrent de quelle manière l'interactant en milieu exolingue déploie des efforts cognitifs, discursifs, imaginatifs, pour comprendre ou se faire comprendre par divers procédés. Comparer les situations d'apprentissage en milieu guidé ou non guidé n'est pas aisé tant les variables sont peu contrôlables (l'individu observé en classe a-t-il un contact en milieu ouvert avec la langue étudiée ou non ?). Les concepts proposés par 
la linguistique étudiant la communication exolingue ont permis de décrire en partie les échanges en classe : la présence du professeur, qui guide l'apprentissage, qui assure la 'médiation'entre la langue, le public et les activités d'enseignement, est un élément incontournable.

\subsection{Une action à accomplir}

Dans une situation de classe de langue, il est exigé des participants d'accomplir une suite d'actions verbales, dans un certain ordre, et selon une certaine méthode. L'occurrence d'activités didactiques ${ }^{3}$ distingue radicalement l'appropriation en milieu guidé de l'acquisition en milieu ouvert ${ }^{4}$. C'est l'enseignant, médiateur du processus d'apprentissage, qui met en place l'activité didactique, que l'on peut définir comme une activité langagière se déroulant selon un certain protocole, proposé par le manuel ou par l'instructeur, et demandant aux usagers de fournir un notable effort cognitif. Le schéma d'une activité didactique est reconnu des participants ; à défaut l'enseignant en rappelle le fonctionnement, en donne la légitimité, et expose la manière de le réaliser, un peu comme on exposerait les règles d'un jeu. Voyons comment, dans la séquence ciaprès ${ }^{5}$, l'enseignant lance une activité de lecture, en annonçant le travail à faire, en précisant la localisation de l'exercice sur le livre et en désignant l'exécutant (corpus Rivière) :

P. /.../ nous allons travailler sur le premier exercice/petites annonces/d'accord ?/ qui est en HAUT/de la page/soixante douze/petites annonces/qui veut lire/la petite annonce numéro 1 ?//est-ce qu'il y a quelqu'un qui veut bien LIRE ? qui est-ce qui peut LIRE/ceci ? l'annonce/la petite annonce numéro un?/ Masako/vous voulez bien lire? la petite annonce numéro un ?//s'il vous plaît?

L'apprentissage est comme formaté par l'activité didactique proposée. L'enseignant fournit les instructions nécessaires pour que les tâches puissent être réalisées. Aussi le discours de la classe est-il émaillé d'injonctions (nous allons faire des exercices de révision, vous allez vous présenter devant le groupe...) ou de questions poussant les apprenants à devenir des protagonistes de l'interaction didactique. La séquence qui suit nous permet de voir que l'activité didactique elle-même peut être l'objet de l'appropriation. Le professeur fait chercher son intitulé (compréhension orale) qu'il fait associer à un contenu (écouter des cassettes, parler des actualités), afin de constituer, semble-t-il, une culture scolaire par la sensibilisation aux actions à accomplir dans ce cadre (corpus Bigot).

$\mathrm{P}$ voilà vous écoute $z$ des cassettes donc qu'est-ce que vous faites vous essayez de: comment ça s'appelle ça l'activité qui quand on écoute des cassettes comment ça s'appelle ça on fait de la A1 XXX $P \quad$ de la quoi $\uparrow$ A2 oral peut-être

P ouais de la quoi de la hm hm oral qu'est-ce que vous essaye $z$ de faire A3 compréhension $\mathrm{P}$ compréhension orale ok (elle écrit au tableau) donc avec Marie Berger XXX le lundi matin vous parlez des actualités vous faites de la compréhension orale.

L'activité didactique est le vecteur obligé de l'appropriation. La consigne, sous la forme d'un dire de faire, constitue un discours intermédiaire entre l'activité demandée et sa 
réalisation par les partenaires apprenants. L'appropriation en classe passe par l'effectuation de ces activités et par la compréhension de la tâche à accomplir.

2.2. L'enseignant comme médiateur

20 La mise en place d'une activité requiert de la part de l'enseignant la coordination simultanée de plusieurs éléments. C'est particulièrement probant lors de la mise en train d'une activité pédagogique où l'enseignant se charge de désigner le locuteur, de décrire la tâche à accomplir, d'indiquer le support, l'item sur lequel on travaille. Dans l'extrait suivant, le professeur s'adresse à une étudiante japonaise débutante et lui donne une série d'injonctions et de conseils sur la manière dont il faut faire un exercice (corpus Rivière).

\begin{tabular}{|l|l|l|}
\hline $\mathrm{P}$ & $\begin{array}{l}\text { alors Yukari la phrase un } \\
\text { Yukari un } \uparrow\end{array}$ & désigne l'étudiante et pointe l'énoncé \\
\hline $\mathrm{P}$ & $\begin{array}{l}\text { la phrase un/on te donne vous + } \\
\text { le verbe parler donc tu dois poser des questions } \\
\text { Yukari mm }\end{array}$ & \\
\hline $\mathrm{P}$ & $\begin{array}{l}\text { avec la langue stan-dard d'accord : } \\
\text { ça veut dire avec EST-ce que + } \\
\text { Yukari mm mm }\end{array}$ & \\
\hline \hline $\mathrm{P}$ & $\begin{array}{l}\text { indications métalinguistiques l'action à accomplir } \\
\text { et avec la phrase affimative + } \\
\text { mais XXX tu me montes la voix XXX } \\
\text { une phrase interrogative : alors vas-y } \\
\text { Yukari (rires) }\end{array}$ & \\
\hline \hline $\mathrm{P}$ & indications sur la forme et l'intonation \\
\hline \hline
\end{tabular}

21 On observe un véritable morcellement des actions à accomplir. Est indiqué à une étudiante de niveau débutant, sur quelle phrase elle doit porter son attention, ce qu'elle doit faire (poser une question), quelle forme elle doit utiliser. Il convient d'explorer ce qui est exigé de l'apprenant en observant attentivement le lien entre l'injonction faite par l'enseignant et les réponses fournies. L'activité cognitive d'un apprenant est le plus souvent cachée à l'observateur, car la tâche pédagogique proposée à la collectivité n'est accomplie de manière explicite que par quelques-uns des participants. Notons que l'apprenant fait parfois des commentaires sur ce qu'il fait, et que l'on a ainsi in situ une trace des opérations d'apprentissage, trace que l'on peut aussi trouver lorsque il y a tenue d'un journal de bord ou lors d'entretiens avec les apprenants. Entre la matière à apprendre, le maniement du support et l'appropriation, l'enseignant inscrit sa médiation par la progression qu'il contrôle en partie, par l'évaluation qu'il exprime : il joue un rôle de régulateur de l'appropriation. 
22 Mais si une attention particulière doit être accordée au guidage des opérations langagières, il ne faut pas que soient oubliés les moments - furtifs - où l'apprenant s'exprime à propos de son propre apprentissage. L'apprenant reste d'une certaine manière le maître de la façon d'apprendre et d'interagir. Inattention, ou attention à des événements fortuits, refus de participation ou retour à l'activité de classe sont des comportements qui font partie du quotidien d'un apprentissage en classe.

2.3. Apprendre avec les autres (ou par les autres)

23 Apprendre une langue au sein d'un groupe veut dire que l'on est engagé dans le dialogue entre les membres du groupe et qu'il y a exposition discursive plurielle: exposition aux dires du professeur, rencontre avec des textes, avec des traces écrites, mais aussi écoute des dires des autres apprenants. L'interpénétration et la diversité des discours entendus ou produits rend difficile à établir le lien entre les objets discursifs rencontrés et les données saisies. Qu'apprend-on des tentatives d'un pair, quelle incidence a le fait d'être le témoin des essais et des corrections données à un compagnon? (voir Nussbaum, 1999). On a l'habitude de prendre comme facteurs déterminants pour l'apprentissage les instructions données par l'enseignant, les contenus proposés dans les programmes, mais plus rarement invoque-t-on les conséquences potentielles d'une confrontation à des dialogues entre un expert et des candidats apprenants. Les participants sont là pour apprendre, mais ils n'en sont pas moins fréquemment amenés à définir leur identité, à indiquer les modalités de la rencontre et, comme le montre Bigot (2001) dans sa recherche, la construction de la relation interpersonnelle s'établit lors de moments de contact privilégiés (début de cours, difficultés longtemps occultées dans l'interaction en classe). Ce que l'apprenant dit de lui, comment il interagit dans la présentation de soi, comment il relate des éléments biographiques, sont des données qui contribuent à la construction de son identité et à son rapport aux autres participants, et peut-être aussi à l'apprentissage.

Dans une classe :

- les apprenants sont confrontés à des formes interactionnelles dans lesquelles il y a à la fois asymétrie (entre l'enseignant et eux-mêmes) et relative symétrie (entre apprenants), asymétrie qui n'est pas nécessairement préjudiciable, si on se souvient que le monde social oblige constamment l'individu à être engagé dans une interaction où toute forme conversationnelle se fait dans la reconnaissance du rôle ou de la place de l'autre.

26 - il s'agit d'une prise de parole publique, qui s'apparente à une parole devant un auditoire (différant en cela de la conversation ordinaire), et à ce titre, l'interaction a un effet sur les affects et l'image que l'individu se fait de lui-même. Ainsi, faire une erreur devant une assistance, ou la faire plusieurs fois de suite, met en jeu des émotions qui peuvent avoir un effet sur les facteurs cognitifs. Ne peut-on imaginer tel participant préférer ne jamais risquer de prendre la parole pour ne pas s'exposer au déshonneur public d'une erreur! Mais un effet contraire est aussi imaginable.

La classe, lieu qui se veut propice à l'apprentissage, met en mouvement une multiplicité de facteurs qui peuvent, selon les cas, aider ou retarder l'appropriation. Le désir d'apprendre, le désir d'être le meilleur du groupe, un programme adapté à son propre rythme, le rapport à la langue apprise, l'aptitude à développer une compétence orale ou écrite, l'occasion d'entendre la langue à l'extérieur ou pas, l'impact du professeur, l'interinfluence entre les apprenants, autant de facteurs qui jouent des rôles 
déterminants mais dont la pondération risque fort d'échapper au chercheur... tout comme à l'usager lui-même.

3. Quelques perspectives de recherche à propos de pratiques de transmission de savoir

Mieux connaître l'appropriation en classe de langue demanderait à ce que l'on soit à même de répondre aux questions suivantes : que se passe-t-il effectivement en classe, quels sont les événements de la classe qui entourent les actes d'appropriation, quel processus instructionnel est mis en place et quelle influence exerce-t-il sur l'appropriation, comment le schéma d'action est-il reconnu des élèves? Mais on risque fort d'avoir le plus grand mal à déterminer les éléments qui, dans le dispositif instructionnel, sont déterminants et favorisent effectivement l'apprentissage. Lorsqu'on est face à un groupe et non à des individus, la question de l'appropriation se pose de toute autre manière, car comment dissocier les trajectoires d'appropriation, qui sont individuelles, du moins en partie? Les propositions de D. Allwright (1988), rappelant l'impasse dans laquelle se trouvent des recherches qui voudraient prouver de façon globale quelle est la meilleure méthode, vont dans le sens d'un développement de recherches à spectre moins large. Traiter les questions de façon plus circonscrite permettrait des avancées moins ambitieuses mais plus sûres. On peut espérer parvenir, pour un contexte donné, à répertorier les événements et les actes de parole qui se produisent dans une classe. Dans cette perspective, plus prudente ou plus réaliste, nous proposons quelques pistes autour de la notion de pratique de transmission et de l'intérêt qu'il y aurait à consigner les pratiques, afin de mettre au jour la singularité d'un travail sur la langue s'accomplissant dans les contextes d'enseignement.

\subsection{Les pratiques de transmission en classe de langue}

On peut considérer qu'au sein des cultures, les pratiques de transmission sont un métissage entre des traditions héritées et des modes communicatifs survenant avec la modernité ou le renouvellement de pratiques pédagogiques. Les institutions éducatives sont le vecteur de ces pratiques : observer le déroulement d'une interaction en classe, les discours produits, permet d'avoir accès à une partie des pratiques dites de transmission. On entend ici par pratiques de transmission les pratiques langagières didactiques (verbales, non verbales, mimogestuelles) et les pratiques interactionnelles qu'un expert met en œuvre afin qu'un public moins savant puisse s'approprier des savoirs et des savoir-faire. Ces pratiques dépendent de la culture d'origine des interactants, de la formation de l'enseignant, de son expérience, et de sa personnalité. Ainsi des actions aussi diverses que les façons de donner la parole (ou ne pas la donner), de se mouvoir dans l'espace de la classe, de faire appel à la mémoire des élèves, de les faire participer au processus de découverte du sens, d'autoriser ou non les improvisations, d'avoir recours à des exemples, de faire des oppositions entre les termes, des comparaisons, etc., sont autant de facettes d'une pratique de transmission. Les activités didactiques formalisées, inscrites dans une tradition éducative donnée (traductions, commentaires de textes, jeux, etc.), aussi bien que des pratiques pédagogiques plus libres, font partie des pratiques de transmission. Ces manières de faire - mêlant stratégies et tactiques - diffèrent selon les contextes et les personnes. Les usagers peuvent avoir à leur disposition des matériaux identiques (par exemple le contenu d'une méthode) mais inventer des modes de réalisation qui leur sont propres. De Certeau (1990, p. 59) distingue la stratégie de la tactique :

J'appelle stratégie le calcul (ou la manipulation) des rapports de force qui devient possible à partir du moment où un sujet de vouloir et de pouvoir (une entreprise, une armée, une cité, une institution scientifique) est isolable. 
30

qu'il y ait stratégie, il faut qu'il y ait intention stratégique, choix entre plusieurs possibles, calcul. La tactique ne se donne pas de projet global, elle fonctionne au coup par coup, elle profite des occasions, se faufile, s'apparente à la ruse. La classe de langue voit la mise en place de pratiques qui se situent entre la stratégie, connue d'avance, planifiée, et la tactique, qui surgit dans le vif de l'échange et pousse les protagonistes à improviser des solutions.

3

On fait l'hypothèse que l'enseignant, pour accomplir sa tâche dispose d'un certain répertoire, qui se constitue progressivement et peut-être par empilement. Le répertoire didactique serait un ensemble hétéroclite de modèles, de savoirs, de situations sur lesquels un enseignant s'appuie. Ce répertoire se constitue au fil des rencontres avec divers modèles (un professeur que l'on a connu, par exemple), par la formation académique et pédagogique, par l'expérience d'enseignement, qui elle-même modifie le répertoire. Lorsqu'il s'agit de l'apprentissage d'une langue, c'est le répertoire verbal tout entier qui peut constituer une des ressources didactiques.

3.2. Pratique linguistique et pratique interactionnelle

Tenter de dépister des traces de ce répertoire d'enseignement fait l'objet d'une recherche collective, dans le cadre d'une recherche amorcée au sein du groupe DELCASYLED à Paris III. Le corpus DELCA (constitué grâce à C. Carlo) rassemble plusieurs séquences de classe de français langue étrangère, au cours desquelles le même enseignant se livre à des activités didactiques différentes. Il s'agit de cours donnés en milieu universitaire à des étudiants assez avancés et résidant en France. L'enseignant, pour réaliser sa performance de professeur, puise à la fois dans son répertoire linguistique ordinaire et fait appel à un répertoire didactique et interactionnel. À titre d'exemple, examinons ce que développe $\mathrm{S}$. (le professeur) comme pratiques de transmission au cours d'un travail de reprise, qui s'effectue à la suite d'exposés d'étudiants. Dans les quelques lignes transcrites qui suivent, il s'agit d'examiner un usage erroné qui a été fait de la majuscule. On sera attentif à l'entrecroisement d'une pratique qui s'attache à la langue, et d'une pratique interactionnelle (fort vive), qui se construit au fil des dires des interlocuteurs-apprenants. 
1S: le deuxième petit point avec FRANCCAIS + avec le mot français quand est-ce qu'on met une majuscule et quand est-ce qu'on ne met pas une majuscule + vous alle $z$ me dire nous on en met jamais + c'est vrai (rire) vous n'en mettez jamais mais quand même il faut une majuscule alors quand est-ce que vous mette $z$ une majuscule + français ou thailandais ou turc +

2 E 1 : au début

3S: au début (rire) tu commenceras beaucoup de phrases avec Français ${ }^{\uparrow}$ (elle écrit au tableau) si + le Général de Gaulle + Français écoutez moi + Français aidez-moi (rire) mais c'est très rare de commencer avec Français c'est juste dans l'exhortation + alors quand est-ce qu'on peut + quand est-ce que français va prendre une majuscule ${ }^{\uparrow}$

$4 \mathrm{E} 2: \mathrm{xx}$

$5 \mathrm{~S}$ : ouais quand on +

$6 \mathrm{E} 3$ : on parle des gens

7S: (elle écrit au tableau) les Français sont + comment je vais terminer ça + les Français sont très $=$

$8 \mathrm{E} 4:=$ peu

$9 \mathrm{~S}$ : très et après $\uparrow$

$10 \mathrm{E} 5: \mathrm{xx}$

$11 \mathrm{~S}:$ les Français sont très français + pas mal ça + avec des guillemets (elle écrit au tableau) + les Français sont très franchouillards vous vous souvene $z \uparrow$

12 E 6 : ouais

$13 \mathrm{~S}$ : vous vous souvenez franchouillards vous avez oublié ${ }^{\uparrow}$ quand on parle des gens + une majuscule mais quand on parle de la langue +qu'est-ce qu'on dit ${ }^{\uparrow}$ le français est $=$

$14 \mathrm{E} 7$ : difficile

$15 \mathrm{E}$ 8: bel

$16 \mathrm{~S}$ : ça c'est de la propagande + quand on parle des personnes majuscule quand on parle de la langue + une minuscule et puis évidemment aussi une minuscule quand c'est l'adjectif

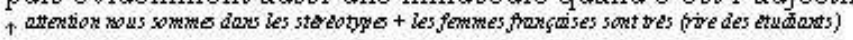
tresquon $=$

$17 \mathrm{E9}:$ = arrogantes

$18 \mathrm{~S}$ : arrogantes ${ }^{\uparrow}$ (rire des étudiants) tu as eu des problèmes avec les femmes françaises mon pauvre xx (rire des étudiants) écoute $z+$ je crois que les femmes françaises sont très arrogantes mais les hommes sont + relativement arrogants aussi

19 E10: c'est moins grave

$20 \mathrm{~S}: \mathrm{hm}^{\top}$

21 E1 : c'est moins grave $\mathrm{xx}$

22 E11: ouais + c'est moins grave

$23 \mathrm{~S}$ : ah bon ${ }^{+}$pourquoi c'est + pourquoi c'est moins grave du côté masculin $\uparrow$

24 E12: ouais parce que $\mathrm{xx}$ (rire des étudiants)

$25 \mathrm{~S}$ : ça c'est int + tu trouves que c'est plus grave les femmes arrogantes ${ }^{*}$ 
26 E9: je dirais prétentieuses

$27 \mathrm{~S}$ : et les hommes dis donc + (rire) ouais + c'est biz + ta ta remarque est bizarre ${ }^{\uparrow}+$ (rire des étudiants) tu as eu des problèmes avec les femmes françaises ma pauvre ${ }^{\top}$

28 E9: un petit peu+oui

$29 \mathrm{~S}$ : vous avez l'air d'avoir eu des TRES mauvaises expériences avec les Français (rire des étudiants) et avec LE français aussi + donc les Français majuscule + la langue française minuscule + si c'est adjectif $+c^{\prime}$ est normal + minuscule ++ mais n'oubliez pas quand même + même si vous ne les aimez pas beaucoup n'oubliez pas que quand vous parlez des GENS + ils MERITENT la majuscule (rire de Set de quelques étudiants) c'est vrai $+c$ 'est important ++ vous ave $z$ un petit peu tendance à penser que les problèmes de majuscules minuscules + les problèmes d'accent + et les problèmes de ponctuation $+c$ 'est pas TRES important hein souvent vous pensez bof c'est pas +1 'orthographe grammaticale ça OUI vous pensez que c'est important mais wous pensez que des petites choses comme ça c'est c'est pas grave + SI c'est aussi un problème d'orthographe ++

L'observation de la pratique langagière de $\mathrm{S}$. déployée dans ce cadre didactique semble indiquer qu'elle prend appui sur plusieurs strates de ressources :

- sur une planification constituée par un programme ou tout élément lié à la préparation du cours. En effet, S. a un projet d'enseignement (ici procéder à une reprise des erreurs faites durant un exposé), ce qui constitue le fil à suivre d'une partie de la séance. La compétence planificatrice la pousse à revenir à son objet lorsqu'elle estime en avoir été éloignée :

TP 1 : le deuxième petit point avec français.

- sur une compétence langagière, qu'elle possède en tant que sujet ordinaire de la langue et en tant que 'usager-professeur', pratique marquée par une capacité à organiser le matériau verbal, à l'expliquer, à le rendre accessible, à procéder à des comparaisons, des récapitulations, etc. comme dans les séquences qui suivent où sont données des normes d'usage de la majuscule :

TP 3 : mais c'est très rare de commencer avec Français c'est juste dans l'exhortation

TP 29: donc les Français majuscule + la langue française minuscule + si c'est adjectif + c'est normal + minuscule + + mais n'oubliez pas quand même +

- sur une connaissance du groupe, à la fois linguistique (les erreurs qu'elle prévoit) et humaine :

TP 29 : vous avez un petit peu tendance à penser que les problèmes de majuscules minuscules + les problèmes d'accent + et les problèmes de ponctuation + c'est pas TRES important hein souvent vous pensez bof c'est pas + l'orthographe grammaticale ça OUI vous pensez que c'est important mais vous pensez que des petites choses comme ça c'est c'est pas grave + SI c'est aussi un problème d'orthographe ++

41 ou la remarque suivante, plus malicieuse :

TP 29 : vous avez l'air d'avoir eu des TRES mauvaises expériences avec les Français

- sur une disposition à savoir mobiliser des situations de la vie ordinaire ou des exemples tirés de la culture, de l'histoire, pour les relier aux mots dont elle veut parler : 
Dans une perspective ethnométhodologique, le chercheur, collecteur de données et transcripteur, peut espérer apprendre de quelle manière les différents participants mettent en place des méthodes pour parvenir à tel but communicatif. On prêterait volontiers à l'enseignant des intentions planificatrices - il sait où il va, il suit un programme -, mais à le suivre dans la confrontation constante avec le dire de l'autre, on découvre qu'il a aussi recours à la ruse, à la nécessité d'inventer sur le champ, de faire avec, d'imaginer des solutions dans l'immédiateté de l'échange. Les participantsapprenants, eux, peuvent être considérés comme des savants non conscients de l'être ou des informateurs - car ils contribuent à la connaissance des modes d'appropriation qui sont les leurs. Il n'est pas rare qu'au sein de l'interaction ils produisent eux-mêmes des commentaires à propos de leurs manières de faire, de leurs difficultés ou de leurs astuces pour résoudre les problèmes. Ils sont alors susceptibles de donner au chercheur-observateur des clefs pour comprendre les modes d'appropriation. Ainsi à la question d'un enseignant «comment as-tu fait?» un étudiant répondait: «instinctivement ». Curieusement un grand nombre de questions que pose l'enseignant au cours de l'interaction commencent par pourquoi, y compris lorsque c'est la bonne réponse qui est donnée. Il se peut que l'enseignant veuille s'assurer de la stabilité du processus acquisitionnel: l'apprenant a-t-il donné la bonne réponse par hasard, ou parce qu'il en connaissait la raison? Comme s'il y avait chez l'enseignant une représentation du processus d'acquisition comme quelque chose qui doit être explicite - ou explicité -, et que l'apprenant peut ou doit verbaliser.

Pour conclure...

48 La classe de langue souffre de bien des maux : elle est talonnée par un extérieur avec lequel elle ne peut jamais rivaliser; la multiplicité des événements et des sources expositives du savoir gomme la possibilité d'établir une relation entre les instructions et le processus appropriatif; aucune méthode ou méthodologie n'a encore fait la preuve qu'elle donnait de meilleurs résultats qu'une autre. Mais envisagée comme un genre interactionnel spécifique, avec ses modes constitutifs, ses contraintes, ses contextes, ses acteurs, considérée comme lieu social, elle devient alors le lieu de déploiement de stratégies, de méthodes, de modes interactionnels, dont l'approche permet de mieux saisir la pluralité des événements qui entourent, escortent l'appropriation d'une langue enseignée, ainsi que la manière dont les interactants s'y prennent pour arriver à leurs fins.

49 Certes, on peut espérer que le développement de recherches à partir de l'observation des classes de langue permettra de mieux pouvoir répondre à des questions que se pose tout enseignant ou tout usager de la classe : quels sont les acquis langagiers que n'avait pas tel apprenant et qu'il a maintenant grâce à un guidage instructionnel, grâce à la mise en place de tel programme et tels contenus, grâce aux types de discours, aux explications fournies, aux types d'interactions encouragées. Pour ce faire, il faut 
engranger, noter, inventorier des pratiques dans leur variété et leur multiplicité. Relisons ce que de Certeau écrit à propos des jeux dans le tome 1 de L'invention du quotidien. Dans toute société, il existe des "jeux», il existe aussi des lieux de remémoration qui permettent de décrire et d'engranger "les coups" (les comptes rendus sportifs par exemple). Ces coups, lorsqu'ils sont connus et racontés se transforment en stratégies identifiées, reproductibles, nommées.

Ne peut-on alors aussi considérer que la classe de langue donne lieu, de la part de ses usagers, à des pratiques, spontanées ou calculées, inventées ou reproduites, qui sont intermédiaires entre la planification qu'exige une stratégie et la ruse qui est saisie au vol de l'occasion? L'analyste ou l'observateur ou encore le formateur, identifiant les coups par la lecture de ce qui se passe dans la classe (les façons de faire) parviendrait à travers l'engrangement des pratiques, à faire l'inventaire des répertoires, des pratiques discursives qui ont pour visée la transmission de savoir-dire ou de savoir-faire en langue étrangère. Reste ensuite à imaginer comment améliorer la rencontre entre professeurs de langue, formateurs, théoriciens, de façon à travailler de concert à la signification de ces pratiques.

\section{BIBLIOGRAPHIE}

ALI BOUACHA, A. 1984. Le discours universitaire, la rhétorique et ses pouvoirs. Peter Lang, Berne.

ALLWRIGHT, D. 1988. Observation in the Language Classroom. Longman, London, New-York.

BAILEY, K.M. 1983. Competitiveness and Anxiety in Adult Second Language Learning : Looking AT and THROUGH the Diary Studies. In Long and Seliger (Dir), Classroom Oriented Research in Second Language Acquisition. Newbury House, Rowley, Massachusetts.

BANGE, P. 1992. A propos de la communication et de l'apprentissage en L2, notamment dans ses formes institutionnelles. In Acquisition et Interaction en Langue Etrangère n ${ }^{\circ}$ 1, 53-86.

BIGOT, V. 2001. Itinéraire d'une recherche sur les interactions verbales en classe de langue : la didactique au détour des chemins. Communication à la Journée Doctorale 01, DEA de Didactologie des langues et des cultures, Université Paris III.

CICUREL, F. \& E., BLONDEL (Dir), 1996. La construction interactive des discours en classe de langue. In Les Carnets du Cediscor $n^{\circ} 4$.

CICUREL, F. 2001. Quand le français professionnel est l'objet de l'interaction. In Les Carnets du Cediscor $\mathrm{n}^{\circ}$ 7, 21-36.

CICUREL, F. et D. VERONIQUE (Dir) à paraître. Discours, action, appropriation. In Actes du XIIe Colloque "Usages pragmatiques et acquisition des langues" 1999. Presses de la Sorbonne nouvelle.

COSTE, D. 1986. Constitution et évolution des discours de la didactique du français langue étrangère. In Etudes de linguistique appliquée $n^{\circ}$ 61, 52-66.

De CERTEAU, M. 1990. L'invention du quotidien, Gallimard, coll. Folio Essais. 
FLANDERS, N. A. 1960. Interaction Analysis in the Classroom : A Manual for observers, University of Michigan Press, Ann Arbor.

GERMAIN, C. 1994. Analyse conversationnelle et structure hiérarchique d'une leçon de langue étrangère. In Les Carnets du Cediscor $n^{\circ} 2,17-26$.

GRANDCOLAS, B. 1994. Champ bibliographique et pistes de lecture. In Actes de la journée d'étude du 29 novembre 1994 "Les journaux de bord d'apprenants de langues, objets de recherches et de questionnements théoriques", Groupe Jan Comenius, Université Paris X Nanterre, 7-14.

KERBRAT-ORECCHIONI, K. 1992. Les interactions verbales. Armand Colin, Paris.

LAMBERT, M. 1994. Les profils d'apprenants comme mode de description et d'explication à la variabilité des apprentissages en langue étrangère. In Acquisition et Interaction en Langue Etrangère $n^{\circ} 4,81-107$.

MEHAN, H. 1979. Learning Lessons : social organisation in the classroom. Harvard University Press, Cambridge, Massachussetts

MOIRAND, S. 1974. Audio-visuel intégré et communication (s). In Langue française $n^{\circ}$ 24, 5-26.

MOIRAND, S. 1986. Décrire les discours d'une revue sur l'enseignement des langues. In Etudes de linguistique appliquée $n^{\circ} 61,27-38$.

MONDADA, L. 2001. Apports de l'éthnométhodologie et de l'analyse conversationnelle à la description de l'acquisition dans l'interaction. In Marquillo-Larruy M., Questions d'épistémologie en didactique du français, Les Cahiers Forell, Université de Poitiers.

NUSSBAUM, L. 1999. Emergence de la conscience langagière en travail de groupe entre apprenants de langue étrangère. In Langages $n^{\circ}$ 134, 35-50.

PORQUIER, R. 1984. Réseaux discursifs et énonciatifs dans l'enseignement/ apprentissage des langues. In LINX n 11, Université Paris X.

SMITH, P.D. 1970. A comparison of the Cognitive and Audiolingual Approaches in Foreign Language Instruction : the Pennsylvania Project. Center for Curriculum Development, Philadelphia.

SOUCHON, M. (Dir) 1998. Pratiques discursives et acquisition des langues étrangères. Université de Franche-Comté, Besançon.

VÉRONIQUE, D. 1994. Quel profil d'apprenant ? Réflexions méthodologiques. In Acquisition et Interaction en Langue Etrangère $n^{\circ} 4,108-130$.

\section{NOTES}

1. Mondada, 2000 souligne l'intérêt que l'on doit avoir pour les « détails » enregistrés et transcrits : « ces détails ne peuvent pas être imaginés mais seulement observés in situ » (p. 113).

2. Nous remercions V. Bigot, C. Carlo, V. Rivière, du DELCA-SYLED de nous avoir permis de travailler sur les corpus qu'elles ont recueilllis.

3. Activité didactique est défini par Germain, 1994 comme l'unité d'analyse de l'enseignement (compréhension orale, explication de vocabulaire, par exemple). Les acteurs d'une situation de classe ont des représentations savantes ou spontanées de l'activité proposée. 
4. Voir plus loin la spécificité du travail sur la langue qui s’accomplit dans le cadre d'une classe

5. Les extraits sont issus de transcriptions faites au sein du DELCA. Le code de transcription varie légèrement d'un transcripteur à l'autre :

$\mathrm{TP}=$ tour de parole.

$+\mathrm{ou} / \mathrm{ou} / /=$ pause. MAJUSCULE $=$ mot ou syllabe accentué. $:=$ allongement syllabique. 'ou $?=$ intonation montante. $\mathrm{X}-\mathrm{X}-\mathrm{X}=$ syllabation. mots soulignés $=$ chevauchement. $\mathrm{XXX}=$ séquence inaudible. Le signe égal $=$ séquences qui se suivent.

\section{RÉSUMÉS}

Cet article trace le chemin parcouru dans la compréhension des discours et échanges se produisant dans une classe de langue : depuis une époque (les années SGAV) où on la présentait surtout comme le lieu de réalisation d'une méthodologie d'enseignement, la classe est progressivement devenue le lieu d'observation d'interactions complexes entre les participants, dont on dégagera ici quelques traits. Etre exposé à la langue dans le cadre d'une classe comporte des points communs : un processus instructionnel programmé, des routines pédagogiques, une organisation de la parole et de la communication. À travers des pratiques qui allient stratégies et ruses, celui qui enseigne puise dans son répertoire les ressources nécessaires au maintien de l'équilibre entre planification et interaction.

This article traces the development in our understanding of foreign language classroom discourse and interaction. Classroom exchanges were first viewed only in relation to the (audiovisual) methodology used, but the focus of attention has since been more and more centred on the complex interaction between the various participants. We identify several important variables in this process : prescribed syllabus, lesson planning, pedagogical routines, improvisational performance, and conclude that the teacher's personal repertoire forms a combination of strategies and tactics which allows him/her to mobilise the necessary resources to strike a balance between planned and spontaneous exchanges.

INDEX

Mots-clés : activité didactique, guidage, interaction didactique, pratique de transmission, répertoire

\section{AUTEUR}

FRANCINE CICUREL

Université Paris III - Sorbonne nouvelle, DELCA-SYLED. fcicurel@club-internet.fr 